\title{
Short-term deleterious effects of standard isolation and cultivation methods on new tropical freshwater microalgae strains
}

\author{
M. Magdalena Aray-Andrade ${ }^{1,2,3 *}$, Miguel I. Uyaguari-Diaz ${ }^{4,5}$ and \\ J. Rafael Bermúdez ${ }^{1,6, *}$ \\ ${ }^{1}$ ESPOL Polytechnic University, Escuela Superior Politécnica del Litoral, ESPOL, Plankton \\ Laboratory, Facultad de Ingenería Marítima, Ciencias Biológicas, Oceánicas y Recursos \\ Naturales, Campus Gustavo Galindo, Guayaquil, Ecuador \\ 2 Joint Postgraduate VLIR NETWORK Master Program in Applied Biosciences, Biodiscovery, \\ ESPOL Polytechnic University, Guayaquil, Guayas, Ecuador \\ ${ }^{3}$ Escuela de Medicina, Universidad Espíritu Santo-Ecuador, Samborondón, Guayas, Ecuador \\ ${ }^{4}$ British Columbia Centre for Disease Control Public Health Laboratory, Vancouver, BC, Canada \\ ${ }^{5}$ Department of Pathology and Laboratory Medicine, University of British Columbia, Vancouver, \\ BC, Canada \\ ${ }^{6}$ Galapagos Marine Research and Exploration, GMaRE. Joint ESPOL-CDF program, Charles \\ Darwin Research Station, Galapagos Islands, Ecuador \\ * These authors contributed equally to this work.
}

Submitted 6 May 2017

Accepted 11 June 2018

Published 19 July 2018

Corresponding authors

M. Magdalena Aray-Andrade, maray@espol.edu.ec

J. Rafael Bermúdez,

jrbermud@espol.edu.ec

Academic editor

Kenneth Leung

Additional Information and Declarations can be found on page 12

DOI 10.7717/peerj.5143

(c) Copyright

2018 Aray-Andrade et al.

Distributed under

Creative Commons CC-BY 4.0

\section{OPEN ACCESS}

\section{ABSTRACT}

Algae with potential biotechnological applications in different industries are commonly isolated from the environment in order to obtain pure (axenic) stocks that can be safely stored for long periods of time. To obtain axenic cultures, antibiotics are frequently employed, and cryopreservation is applied to preserve standing stocks. However, many of these now standard methods were developed using strains derived from pristine to near-pristine environments and cold to temperate regions. The potential effect of the said methods on the life cycle and biochemical profile of algae isolates from hyper-eutrophic and constant hightemperature tropical regions is not well understood. These effects could potentially render them unsuitable for their intended biotechnological application. In this study, we conducted a genetic characterization (18S rRNA) and evaluated the effect of purification (the use of the antibiotic chloramphenicol, CAP) and cryopreservation (dimethyl sulfoxide; DMSO-sucrose mix and glycerol) on the growth rate and lipid content of three new tropical freshwater algal isolates: Chorella sp. M2, Chlorella sp. M6, and Scenedesmus sp. R3, obtained from the Ecuadorian coast. The genetic and morphological characterization revealed a clear discrimination between these strains. All strains cultured with CAP exhibited a lower growth rate. Subsequent to cryopreservation, Chorella sp. M2, Chlorella sp. M6, and Scenedesmus sp. R3 presented no significant difference in growth rate between the cryopreservants. Further, a significantly higher lipid content was observed in the biomass cryopreserved with glycerol in relation to the DMSO-sucrose, with Chorella sp. M2 and Chlorella sp. M6 having twice as much as they had in the first treatment. These results highlight the relevance of selecting an appropriate method for storage, as the materials used can affect the biological performance of different tropical species, although it is still to be determined if the effects observed in this study are long lasting in subsequent cultures of these algae. 
Subjects Aquaculture, Fisheries and Fish Science, Cell Biology, Environmental Sciences, Freshwater Biology

Keywords Lipids, Growth rate, Antibiotics, Cryopreservation, Microalgae

\section{INTRODUCTION}

The cultivation of wild microalgae under defined/controlled conditions has received considerable interest due to their potential as sources of various compounds that have biotechnological and commercial for the nutraceutical, cosmetic, and pharmaceutical industries (Dogaris et al., 2015; Encarnação et al., 2015; Gellenbeck, 2012; Maurya et al., 2016; Rodrigues et al., 2015). Some microalgae serve as essential raw materials in biofuel production due to their ability to accumulate a high lipid content. Microalgae such as Chlorococcum sp. represents a fast-growing specie (John et al., 2011; Mahapatra \& Ramachandra, 2013; Masojídek et al., 2000), and its lipid content can contribute up to $30.55 \pm 2.65 \%$ of dried biomass (Mahapatra \& Ramachandra, 2013). Some Chlorella species are known to produce lipids such that $10-39 \%$ of dried biomass is lipids (Chiu et al., 2015), showing a huge potential as a lipid sources in biotechnology. Recently, the production of microalgal biomass has been scaled up to large outdoor cultivation systems for the production of biodiesel. High temperatures can severely affect the productivity of these systems (Béchet et al., 2013). The isolation and characterization of high-temperature-tolerant microalgal species is important for mitigating these effects, as has been demonstrated with the temperature-tolerant strain Chlorella sorokiniana (Béchet et al., 2013).

One advantage of industrial algal biomass production is that wastewater can be used as a culture media to produce middle- and high-value by-products. The use of wastewater reduces the use of freshwater, decreases the cost of nutrient addition, and assists in the removal of nitrogen and phosphorus, which otherwise would have been released into the environment (Chiu et al., 2015). Several microalgae have been shown to have high bioremediation potential, such as Chlorella and Scenedesmus (Silkina et al., 2017). A significant barrier to using wastewater is that it has such high levels of nitrogen, phosphorus, and other microbes, that many microalgal species have their growth inhibited by it (Mahapatra \& Ramachandra, 2013; Mara, 2004). An efficient strategy to mitigate the potential negative effects of using wastewater as culture media is the isolation of wild algal strains proliferating in it (Mahapatra \& Ramachandra, 2013). However, to assess the biotechnological potential and applications of microalgal species, the first step in assessing the biotechnological potential of microalgal species is to isolate them from the environment and obtain pure (axenic) stocks that can be stored for long periods of time and reused. Given that an elevated bacterial contamination can be expected during the isolation of fresh strains from wastewater (Mara, 2004), antibiotics such as florfenicol, streptomycin, furazolidone, and specially chloramphenicol (CAP) are often used to eliminate bacteria in the culture media (Campa-Córdova et al., 2006; Lai et al., 2009). CAP is highly effective because it inhibits a variety of aerobic and anaerobic microorganisms. However, intensive use of antibiotics in households, agriculture, and 
aquaculture has shown adverse ecological effects, including the development of resistant bacterial populations (Lai et al., 2009), which may require a higher concentration of antibiotics for their elimination from isolated strains. It has been reported that antibiotics can negatively affect the growth of certain algal species, such as Chlorella pyrenoidosa, Isochrysis galbana, and Tetraselmis chui (Lai et al., 2009) which are cultured with CAP.

The cryopreservation of microalgae stocks for long-term storage is important, as it eliminates the requirement to maintain continuous batches of growing strains (Bui et al., 2013). Although the mechanisms involved in cryopreservation are generally understood, the impact is not (Bui et al., 2013) especially on difficult-to-freeze algae isolated from tropical environments (Fuller, Lane \& Benson, 2005). Cryopreservation can cause the formation of ice crystals in the cellular cytoplasm during the freezing process, which disrupt the cell membrane (Bui et al., 2013). In order to reduce this effect, cryopreservants can be added to the culture media to protect eukaryotic cells from the damage caused by freezing. For instance, dimethyl sulfoxide (DMSO), methanol, and glycerol are cell-wall-permeable cryoprotectants. Another cryoprotectant, sucrose, does not permeate the cell membrane and thus its protection is extracellular (Bui et al., 2013; Hubálek, 2003). While the cryopreservant may protect the cell, it also may change the original biochemical profile of the algae through the alteration of their genetic structure and/or metabolic pathways (Müller et al., 2007), making them unsuitable for their intended biotechnological application, for which they were originally isolated.

A crucial step in the potential use of tropical algae as a biotechnological resource is determining the potential effects of commonly employed methods on their biochemical profiles and productivity rates. Thus, the aim of the present study was to evaluate the effect of traditional isolation and cultivation processes used to obtain axenic cultures (treatment with chloramphenicol) and their cryopreservation (DMSO-sucrose and glycerol) on the growth rate and lipid content of four new algae isolates obtained from wastewater in the tropical Ecuadorian coast region.

\section{MATERIALS AND METHODS}

\section{Isolation, identification, and preparation of stocks}

For this study, microalgae strains were isolated from green standing water bodies $(20 \mathrm{ml})$ located around the urban areas of Guayaquil, Ecuador. From each water sample collected, serial 1:10 dilutions were made in $1.5 \mathrm{ml}$ eppendorf tubes using Guillard's f/2 medium (Guillard \& Ryther, 1962) as the culture media. The medium was made using filtered freshwater instead of saltwater.

The strains, identified with morphological differentiation, were as follows: Chorella sp. M2, Chlorella sp. M6, and Scenedesmus sp. R3; Wehr \& Sheath's (2003) method was followed.

The isolated and identified strains were further cultivated in batch cultures with Guillard's f/2 medium, under controlled laboratory conditions (culture cabins at $30{ }^{\circ} \mathrm{C}$ and a $12 / 12 \mathrm{~h}$ photoperiod), in order to obtain sufficient biomass to start the experiments. 


\section{Antibiotic assays}

In order to obtain an axenic culture, CAP was added to the culture media at a final concentration of $50 \mathrm{mg} \mathrm{l}^{-1}$, as recommended by Lai et al. (2009). This concentration was selected because the strains were isolated from hypereutrophic stagnant ponds located around urban areas of Guayaquil; therefore, they were expected to contain an elevated bacterial load.

The experiment to determine the effect of the antibiotic on growth rate comprised three 48-h phases: (1) before the addition of antibiotics, (2) with antibiotics, and (3) after the removal of antibiotics. The removal was done by replacing the culture media with another culture media without using an antibiotic three times in order to rinse off the remnants. Microalgae strains were cultivated in Guillard's $\mathrm{f} / 2$ medium in triplicates on a semi-continuous batch set up over 6 days at $30{ }^{\circ} \mathrm{C}$ and a photoperiod of 12:12. After every growth phase, new culture media were supplied to each bottle to eliminate the bottle effect.

The cell number of each culture, expressed as cells per milliliter (IOC-UNESCO, 2010), was determined after each phase of the experiment using a Neubauer chamber and compound microscope. The growth rate, expressed as $\mu$, was determined by calculating the counting difference between the last and first day of culture divided by the total number of days required by the culture; further, the growth rates of each phase of the experiment were compared.

\section{DNA extraction, polymerase chain reaction, cloning, and sequencing}

After obtaining the axenic cultures, the algae were genetically characterized. Before extraction, to facilitate the disruption of algal cells, eight freeze-thaw cycles were conducted, followed by overnight digestion, using $20 \mu \mathrm{l}$ of $20 \mathrm{mg} \mathrm{ml}^{-1}$ proteinase $\mathrm{K}$ (Qiagen Sciences, Germantown, MD, USA). The DNA was extracted using the UltraClean Soil DNA Kit (MoBio, Carlsbad, CA, USA) following the manufacturer's instructions. The DNA concentration and purity were assessed with Qubit dsDNA high sensitivity in a Qubit 3.0 fluorometer (Life Technologies, Carlsbad, CA, USA) and NanoDrop spectrophotometer (NanoDrop technologies, Inc., Wilmington, DE, USA) respectively. Specific primers targeting V1-V3 regions of the 18S rRNA gene were selected based on previous studies (Amann, Krumholz \& Stahl, 1990; Zhu et al., 2005; Amann, Krumholz \& Stahl, 1990; Zhu et al., 2005). Each $50 \mu \mathrm{l}$ of PCR reaction consisted of $1.5 \mathrm{mM} \mathrm{MgCl} 2$, $0.2 \mathrm{mM}$ nucleotides, $400 \mathrm{mM}$ of each primer, $1.25 \mathrm{U}$ of hot start polymerase (Promega Corporation, Fitchburg, WI, USA), and one $\mu$ l of template DNA. The thermal cycling conditions entailed $94{ }^{\circ} \mathrm{C} \times 5 \mathrm{~min}, 35$ cycles of $30 \mathrm{~s}$ at $94{ }^{\circ} \mathrm{C}, 60 \mathrm{~s}$ at $55^{\circ} \mathrm{C}$, and $90 \mathrm{~s}$ at $72^{\circ} \mathrm{C}$, and a final cycle of $10 \mathrm{~min}$ at $72{ }^{\circ} \mathrm{C}$. PCR amplicons were examined in a $1.5 \%$ agarose $/ 0.5 \times$ TBE gel stained with $1 \times$ GelRed (Biotium, Inc., Hayward, CA, USA) and purified with a QIAQuick PCR Purification Kit (Qiagen Sciences, Maryland, MD, USA) according to the manufacturer's instructions. Subsequently, the amplicons were ligated into pCR2.1-TOPO cloning vectors (Invitrogen, Carlsbad, CA, USA) and transformed into One Shot E. coli DH5 $\alpha$-T1R competent cells following the manufacturer's protocol. At least four transformants per sample were screened for 
inserts following the manufacturer's recommendations. Positive transformants were subsequently grown overnight at $37^{\circ} \mathrm{C}$ in a lysogeny broth with $50 \mu \mathrm{g} \mathrm{ml} l^{-1}$ kanamycin. Plasmids were extracted using the PureLink Quick Plasmid Miniprep Kit (Life Technologies, Carlsbad, CA, USA). A dsDNA high sensitivity kit in a Qubit 3.0 fluorometer was employed for further quantitation assessment (Life Technologies, Carlsbad, CA, USA). Plasmids were end-sequenced in an ABI 3130xl Genetic Analyzer (Life Technologies, Carlsbad, CA, USA) with a M13 forward primer $(-20)$ (5'-GTAAAACGACGGCCAG-3') and M13 reverse primer (5'-CAGGAAACAGCTATGACC-3') using BigDye Terminator version 3.1 cycle sequencing kit (Applied Biosystems, Warrington, UK). The resultant set of DNA sequences were blasted against the SILVA 18S rRNA database (Pruesse et al., 2007).

The partial sequences were deposited into GenBank Chlorella sp. M2 (accession number: MF677855), Chlorella sp. M6 (accession number: MF677856), Scenedesmus sp. R3 (accession number: MF677857).

\section{Phylogenetic tree construction}

To further define the sequences in this study, the sequence alignment of $18 \mathrm{~S}$ rRNA gene was performed with MAFFT multiple aligner and the tree constructed with Jukes-Cantor (Genetic distance model); Method employed: UPGMA with 1,000 iterations (Kearse et al., 2012).

\section{Cryopreservation assays}

In order to determine the effect of cryopreservation on the growth rate and lipid content of the new tropical algal isolates, they were subjected to two treatments, set in triplicates, consisting of glycerol and a DMSO-sucrose mix, respectively. The cultures of Chorella sp. M2, Chlorella sp. M6, and Scenedesmus sp. R3 with concentration in the range of 2-9 $\times 10^{7} \mathrm{ml}^{-1}$ cells were used (Bui et al., 2013). One milliliter of the culture was transferred to $1.8 \mathrm{ml}$ cryovials for the respective treatments. A DMSO and sucrose solution (Fisher Chemical, CSA grade) was added to a final concentration of $10 \%(\mathrm{v} / \mathrm{v})$ and $200 \mathrm{mM}(\mathrm{v} / \mathrm{v})$, respectively (Bui et al., 2013), while glycerol was added to a final concentration of $10 \%(\mathrm{v} / \mathrm{v})$ (Hubálek, 2003) in the corresponding vials. The cryopreservants were added slowly to the cryovials containing the algae as recommended by Bui et al. (2013). Additionally, a negative control comprising cryovials of each microalgae strain without cryopreservants be was prepared.

The cryovials were inserted into a polyethylene container with a screw cap filled with $125 \mathrm{ml}$ isopropanol (Bui et al., 2013). Isopropanol enables a freezing rate of approximately $-1{ }^{\circ} \mathrm{C} \mathrm{min}^{-1}$ (Shiraishi, 2016). This container was stored in a freezer at $-80^{\circ} \mathrm{C}$ for short ( 5 days) and long ( 14 months) periods, respectively, in order to compare the effect of cryopreservation on time growth rate of the algal; the short period was considered because it represents the approximate time required for the replenishment of stocks in semi-continuous cultures with harvest every 5 days; the second was considered to evaluate the storage of stocks for long durations. At the end of each period, the container was removed from the freezer and the cryovials were put to float in a 
beaker filled with water ( $500 \mathrm{ml}$ at $25^{\circ} \mathrm{C}$ ) (Bui et al., 2013), four cryovials at a time. The thawed cell cultures were gently poured into sterile Falcon tubes containing $49 \mathrm{ml}$ of Guillard's f/2 medium at room temperature; it was left unmixed for one $h$ and subsequently carefully inverted five times (Bui et al., 2013). To allow cell recovery after thawing and before the experiments started, the Falcon tubes with the strains were kept at $30{ }^{\circ} \mathrm{C}$ in the dark for $24 \mathrm{~h}$, at dimmed-light for $48 \mathrm{~h}$ and with light with a photoperiod of 12:12 again for $48 \mathrm{~h}$. After this adaptation period, the cultures were transferred to glass vials where they were kept in batch cultures for 6 days before the experiment started. For the experiment, the microalgae were cultivated at $30{ }^{\circ} \mathrm{C}$ and a photoperiod 12:12 for 6 days. At the end of the culture period, the growth rates, expressed as $\mu$, was determined by calculating the counting difference between the last (day 6) and day 1 , and sample for algal lipid content were taken by filtering a volume of $10 \mathrm{ml}$ from each culture using Whatman GF/F and GF/D glass fiber filters $(\sim 0.7$ and $2.7 \mu \mathrm{m}$ pore size, respectively) in accordance to the size of the microalgae.

\section{Total lipid}

The total lipid content from the cryopreservation test was determined following the protocol described by Christie (1994), using a mix of chloroform, dichloromethane, and methanol (1:1:1). The ethereal extract was determined in terms of weight difference (Matos et al., 2016; Prommuak et al., 2012; Yang et al., 2014).

\section{Statistical analysis}

The statistical analysis was performed with analysis of variance within (one-way ANOVA) and between strains (two-way ANOVA) using the Statistica v7.0.61.0 software. The normality of distribution was analyzed with a Shapiro test. A $p$-value of $<0.05$ was considered as statistically significant. Furthermore, a post hoc analysis was conducted with a Tukey test in order to determine the significance. The total lipid was analyzed with nonparametric Kruskal-Wallis and median tests, as the data revealed a non-normal distribution.

\section{RESULTS AND DISCUSSION}

The genetic identification conducted with $18 \mathrm{~S}$ rRNA partial sequences and the SILVA database corresponded with the morphological identification, with the most probable genera being Chorella sp. M2, Chlorella sp. M6, Scenedesmus sp. R3. Moreover, to determine the possible association between our sequences and other characterized algal sequences in the GenBank database, an UPGMA consensus tree was created (Fig. 1A). The consensus sequence percentage revealed a 90\% similarity between M2 and M6 in nucleotide alignment. Sequences from M2 and M6 exhibited a 67.9\% similarity with members of the genera Chlorella. Overall, M2 and M6 clustered with members of the Chlorellaceae family and included genera such as Chlorella and Parachlorella. These results indicate the relationship between members of this family as previously reported (Bock, Pröschold \& Krienitz, 2011; Krienitz et al., 2004). R3 clustered with members of the Scenedesmaceae family, including Coelastrella, Scenedesmus, and 


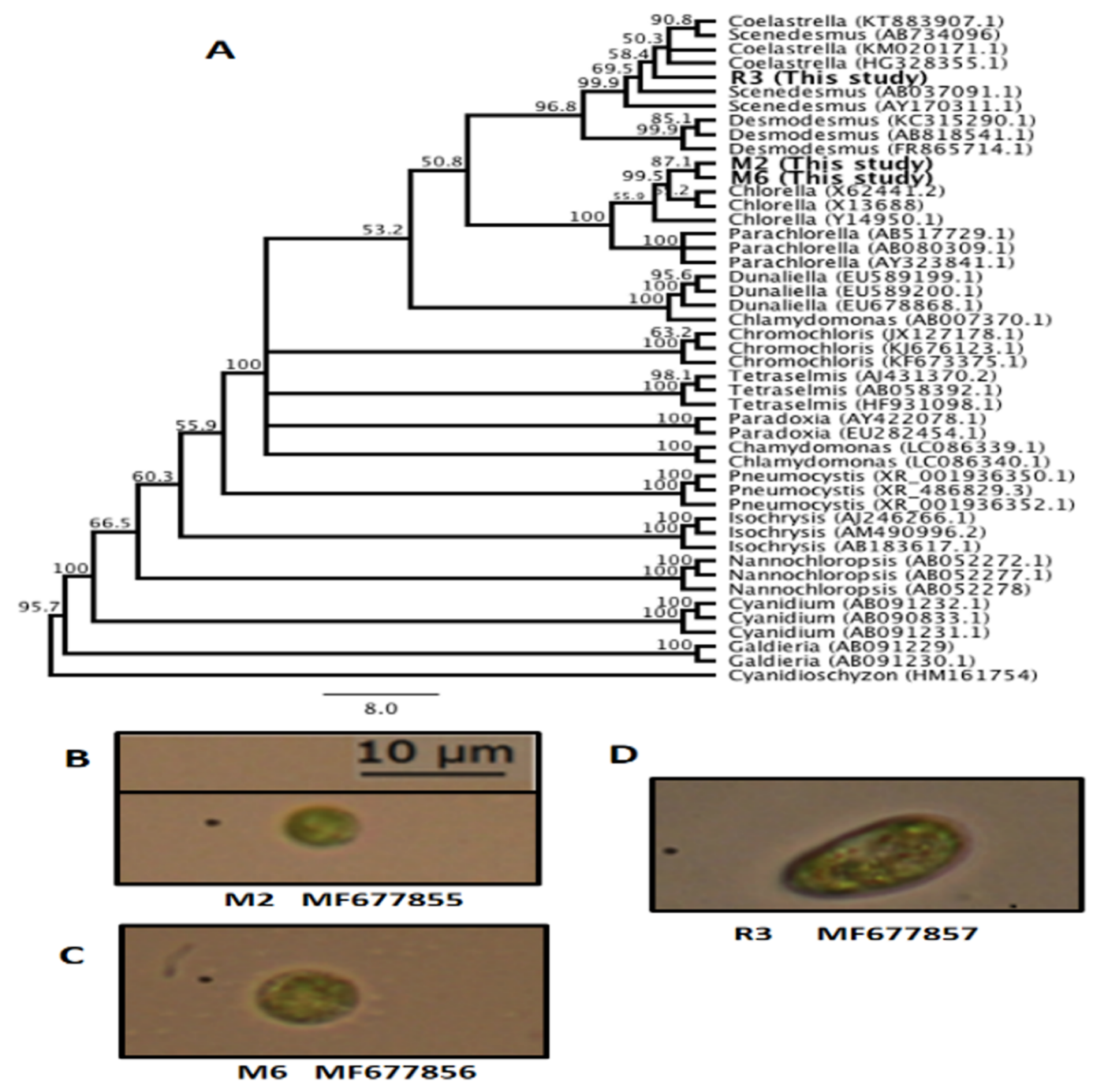

Figure 1 Microalgae isolated in this study. (A) Unweighted pair group method with arithmetic mean (UPGMA) consensus tree of the $18 \mathrm{~S}$ rRNA sequences isolated in this study and other representative algal sequences retrieved from the National Center for Biotechnology Information (NCBI) database. Numbers in the branches represent bootstrap values from 1,000 replications. Numbers in parentheses are the GenBank accession identification numbers. (B) Chlorella sp. M2 MF677855. (C) Chlorella sp. M6 MF677856. (D) Scenedesmus sp. R3 MF677857. Full-size $\quad$ DOI: 10.7717/peerj.5143/fig-1

Desmodesmus (Fig. 1A). The small discrepancies observed in the definition of genera in the clades may be caused due to the partial sequences obtained in this study. Nevertheless, bootstrap values were found to be higher than $50 \%$ for these branches. These findings are also supported by the morphological identification conducted following Wehr \& Sheath's (2003) method for all algal isolates from this study, Chlorella. M2 (Fig. 1B), Chlorella. M6 (Fig. 1C), and Scenedesmus R3 (Fig. 1D).

\section{Growth rate using chloramphenicol}

The microalgae revealed significant differences in growth rate with and without CAP across them (ANOVA, $F=47.99, p=0.0002, \mathrm{~d} f=2$; post hoc in Table 1) and also within 
Table 2 Result of the post hoc statistical analysis of antibiotic treatments.

Before CAP Addition CAP Remove CAP

DMSO-Sucrose

Glycerol

Without

cryopreservants

\section{Chlorella sp. M2}

Before CAP

0.000725
0.020553

0.000725

0.020553

Addition CAP

0.020553

DMSO-sucrose

Glycerol

Without

cryopreservants

Chlorella sp. M6

Before CAP

0.0007049

Addition CAP

Remove CAP

DMSO-sucrose

0.265358

Glycerol

Without

cryopreservants

\section{Scenedesmus sp. R3}

Before CAP

Addition CAP

Remove CAP

DMSO-sucrose

Glycerol

Without cryopreservants

cryopreservants

\begin{tabular}{|c|c|c|c|c|c|c|}
\hline Microalgae & Before CAP & $\begin{array}{l}\text { Addition } \\
\text { CAP }\end{array}$ & $\begin{array}{l}\text { Removal } \\
\text { CAP }\end{array}$ & $\begin{array}{l}\text { DMSO + } \\
\text { sucrose }\end{array}$ & Gly & $\begin{array}{l}\text { Without } \\
\text { cryopreservants }\end{array}$ \\
\hline $\begin{array}{l}\text { Chlorella sp. } \\
\text { M2-Chlorella } \\
\text { sp. M6 }\end{array}$ & 0.522912 & 0.486532 & 0.289684 & 0.000721 & 0.028756 & 0.103303 \\
\hline $\begin{array}{l}\text { Chlorella sp. } \\
\text { M2-Scenesdesmus } \\
\text { sp. R3 }\end{array}$ & 0.020487 & 0.001001 & 0.007372 & 0.001287 & 0.028756 & 0.002993 \\
\hline $\begin{array}{l}\text { Chlorella sp. } \\
\text { M6-Scenesdesmus } \\
\text { sp. R3 }\end{array}$ & 0.006173 & 0.000535 & 0.001764 & 0.623298 & 1.00000 & 0.038330 \\
\hline
\end{tabular}

Notes:

Before, addition and removal of chloramphenicol (CAP); cryopreservation treatments with dimethyl sulfoxide + sucrose (DMSO + S), glycerol (Gly), and without cryopreservants. The values given are $p$ values. Values with $p<0.05$ are presented in bold. 


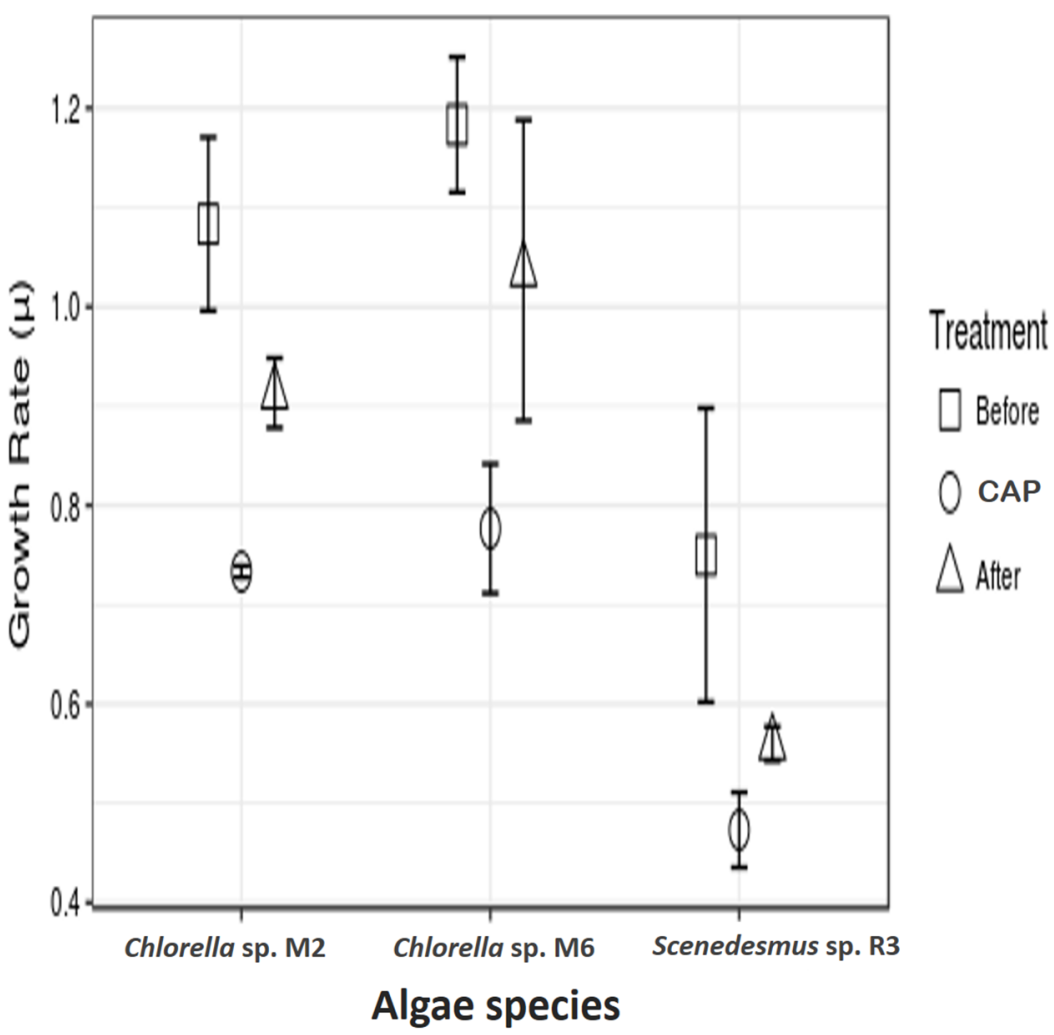

Figure 2 Antibiotic assays. Growth rate of microalgae before the addition of antibiotics, with chloramphenicol in final concentration of $50 \mathrm{mg} \mathrm{l}^{-1}$ (CAP) and after the removal of antibiotics.

Full-size $\operatorname{iv}$ DOI: 10.7717/peerj.5143/fig-2

each strain (ANOVA, Chlorella sp. M2: $F=47.94, p=0.00228$; Chlorella sp. M6: $F=55.96$, $p=0.0017$; Scenedesmus sp. R3: $F=9.84, p=0.034945$; post hoc in Table 2) (Fig. 2). Similar results have been reported by Lai et al. (2009), who found concentrations 20-40 $\mathrm{mg}^{-1}$ of CAP inhibited growth in C. pyrenoidosa, which is close to the range employed in the present study $\left(50 \mathrm{mg} \mathrm{l}^{-1}\right)$. This effect has been attributed to the CAP acting as an inhibitor of photosynthetic oxygen evolution as well as an inhibitor of protein synthesis in chloroplasts, that, in turn, affects the chlorophyll synthesis in photosynthetic organisms. Seoane et al. (2014) reported a decrease in cell size and in chlorophyll $a$ content but detected an increase in chlorophyll $a$ fluorescence in microalga Tetraselmis suecica. This could be due to an inhibitory effect localized on the oxidant side of mitochondria, caused by blockage of the electron transport chain at the photosystem (PS) II level. The inhibition of the electron flow in the PS II reaction center at the donor side provokes a decrease in the chlorophyll $a$ fluorescence, while if the inhibition is produced in the acceptor side of the PS II, an increase in the chlorophyll $a$ fluorescence is observed (Cid et al., 1995).

In addition to the toxic effect of CAP on photosynthesis, the high antibiotic concentration used in the present study eliminated the possibility of the presence of bacteria associated with freshly isolated strains, which could have contributed to the observed negative effect. It is known that microalgae live in synergism with certain 


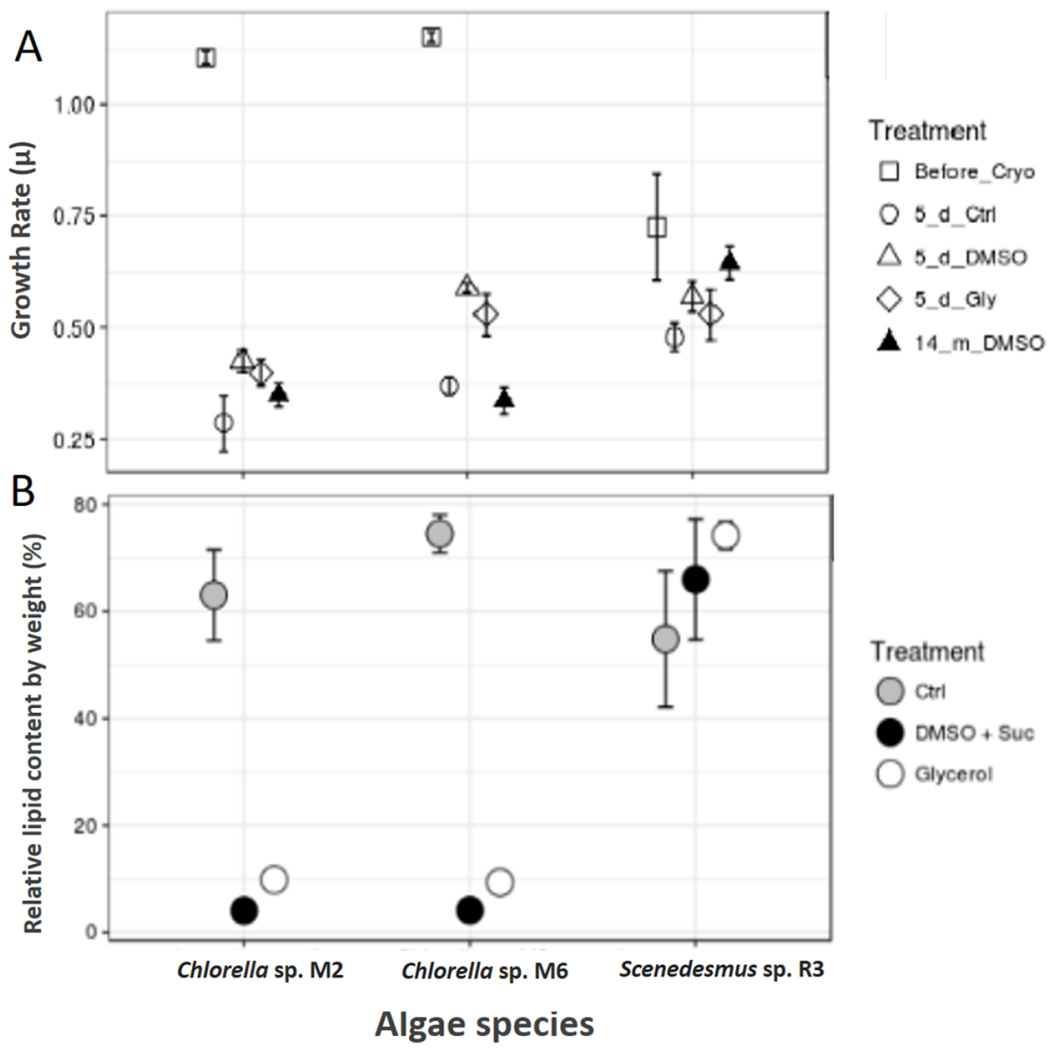

Figure 3 Cryopreservation test and total lipid. (A) Microalgae growth rate before and after cryopreservation during 5 days with dimethyl sulfoxide and sucrose (DMSO), glycerol (Gly), plus a control without cryopreservant (Ctrl) and after cryopreservation during 14 months with dimethyl sulfoxide and sucrose. (B) Total lipids of microalgae, control (Ctrl), after cryopreservation with dimethyl sulfoxide (DMSO + sucrose) and glycerol.

Full-size DOI: 10.7717 /peerj.5143/fig-3

bacteria, interacting with each other through nutrients interchange, signal transduction, genes transference, and other means (Kouzuma \& Watanabe, 2015). Lubarsky et al. (2010) demonstrated that the microalgal biomass was significantly lower in axenic cultures (the antibiotics added) as compared to the cultures containing bacteria. Nevertheless, the two strains of Chlorella demonstrated a higher growth rate in relation to Scenedesmus sp. after the removal of CAP. This demonstrates that some microalgae strains can recover from CAP exposure, such as Chlorella sp. M2 and M6, result observed here and also by other authors, such as Campa-Córdova et al. (2006) and Lai et al. (2009), who employed CAP at concentrations of $0.5 \times 12.0 \mathrm{mg} \mathrm{l}^{-1}$. CAP has been shown to have no significant effect in the growth of C. pyrenoidosa and I. galbana. A more detailed study is necessary to access the actual cause of the observed effect.

\section{Cryopreservation test}

Scenedesmus sp. R3 did not show a difference in its growth rate before and after cryopreservation, unlike the two other strains (ANOVA, $F=3.52$, $p$ 0.097448; post hoc in Table 2) (Fig. 3A). Saadaoui et al. (2016) reported that Scenedesmus cells rapidly recover after 1 month of storage in liquid nitrogen using dimethyl sulfate as 
cryoprotectant. Microalgae exhibited differences in growth rate among different strains (ANOVA, $F=925.02, p<0.001, \mathrm{~d} f=3$; post hoc in Table 1). Our two strains of Chlorella sp. after 14 months of cryopreservation showed a growth rate lower than the strain cryopreserved for 5 days. No microalgal strains showed different growth rates between cryopreservation types; however, the cultures that were frozen without a cryopreservant demonstrated a lower growth rate compared to cultures with cryopreservants; they also required more time for recovery (ANOVA, Chlorella sp. M2: $F=9.44, p$ 0.014015; Chlorella sp. M6: $F=44.77$, $p$ 0.000248; post hoc in Table 2) (Fig. 3A). This may be due to the need for permeable and impermeable cryopreservants such as DMSO-sucrose, because sucrose increases solution osmolarity that causes cell dehydration. Sucrose works synergistically with DMSO to minimize intracellular ice damage by increasing the total concentration of the solute and reducing the amount of ice formed in the cell (Bui et al., 2013). Moreover, glycerol decreases the freezing point of water and biological fluids through colligative action (glycerol/water to a minimum of $-46{ }^{\circ} \mathrm{C}$ ), and it prevents eutectic crystallization (Hubálek, 2003). Scenedesmus sp. R3 exhibited the smallest difference between the growth rates of cultures with cryopreservation and without cryopreservants. A more detailed study is necessary to evaluate the effect of frostbite without cryopreservants.

\section{Total lipid content}

The total lipid content was analyzed in the biomass of microalgae strain using cryopreservants. Scenedesmus sp. R3 showed an increase in the total lipid content after cryopreservation, unlike the other two strains that showed a decrease by a large proportion (Chi square $=5.955556, \mathrm{~d} f=2, p=0.0509$ ) (Fig. 3B). Saadaoui et al. (2016) confirmed that the lipid production could be affected by the cryopreservation method that could result in an increase and decrease in lipid content. When the two cryopreservants were compared, a higher lipid content was observed in the biomass that was cryopreserved with glycerol. Thus, in Chorella sp. M2 and Chorella sp. M6, the relative lipid content was twice as high compared to the biomass cryopreserved with DMSO + sucrose (Chi-square $=6.300000, \mathrm{~d} f=2, p=0.0429)$ (Fig. 3B). This might be due to the fact that some algae can utilize glycerol as a carbon source. Since, Chlorella vulgaris achieved maximum lipid productivity in glycerol supplemented culture medium (Sharma et al., 2016), this could be the reason for the observed increase in lipid content. In addition, the cytosolic lipid bodies increased in number as well as in size as the intensity of the light increased. So, the use of glycerol could advantageous for lipid production.

Tropical microalgae are adapted to high temperatures and sunlight. For this reason, these microalgae can be cultivated outdoors efficiently for lipid production. Wang et al. (2013) showed that lipid and protein content varied with different nitrogen and light regimes. Higher light intensities tend to induce the production of more neutral lipids in comparison to polar structural lipids in algae cells. These tropical freshwater microalgal strains have potential biotechnological applications; therefore, further studies are required on them. 


\section{CONCLUSION}

It was observed that the selected cultivation process can affect the productivity of tropical freshwater microalgae isolates, as chloramphenicol can reduce the growth of microalgae when used at the concentration $50 \mathrm{mg} \mathrm{l}^{-1}$. However, the effectiveness of lower concentrations on the elimination of bacteria from the cultures remains to be demonstrated. It is still necessary to determine if the observed deleterious effect of high CAP concentration in Scenedesmus sp. R3 growth rate is long lasting in the strains when they are cultured subsequently.

Both DMSO-sucrose and glycerol were found to be effective microalgae cryopreservants in most cases, especially with Scenedesmus sp. R3.

The lipid content of the algae was found to be significantly affected by the cryopreservation method. Thus, species such as Chlorella sp. M2 and Chlorella sp. M6 should be cryopreserved with glycerol when they are cultured to obtain a higher lipid gain per cell. With regard to the antibiotics, it is necessary to determine whether the negative effects of DMSO-sucrose on the algal lipid content is temporary or continues in future cultures.

The results highlight the importance of selecting appropriate methods to obtain axenic cultures and their subsequent storage, as these can severely affect the biological performance of different species of tropical freshwater microalgae isolates. Further, whether these effects are long lasting remains to be determined.

\section{ACKNOWLEDGEMENTS}

The authors want to acknowledge Dr. Patricia Manzano and Dr. Washington Cardenas for support with laboratory material, and Viviana Santander for assistance.

\section{ADDITIONAL INFORMATION AND DECLARATIONS}

\section{Funding}

This project was funded by the Dean of Research at ESPOL, by the grant G1-DI-2015. This project was executed within the Join postgraduate VLIR NETWORK Master Program in Applied Biosciences. The funders had no role in study design, data collection and analysis, decision to publish, or preparation of the manuscript.

\section{Grant Disclosures}

The following grant information was disclosed by the authors:

Dean of Research at ESPOL: G1-DI-2015.

Join postgraduate VLIR NETWORK Master Program in Applied Biosciences.

\section{Competing Interests}

The authors declare that they have no competing interests.

\section{Author Contributions}

- M. Magdalena Aray-Andrade performed the experiments, analyzed the data, prepared figures and/or tables, authored or reviewed drafts of the paper, approved the final draft. 
- Miguel I. Uyaguari-Diaz performed the experiments, analyzed the data, contributed reagents/materials/analysis tools, prepared figures and/or tables, authored or reviewed drafts of the paper, approved the final draft.

- J. Rafael Bermúdez conceived and designed the experiments, analyzed the data, contributed reagents/materials/analysis tools, prepared figures and/or tables, authored or reviewed drafts of the paper, approved the final draft.

\section{Data Availability}

The following information was supplied regarding data availability:

The data is available at GenBank, accession numbers MF677855, MF677856, and MF677857.

\section{Supplemental Information}

Supplemental information for this article can be found online at http://dx.doi.org/ 10.7717/peerj.5143\#supplemental-information.

\section{REFERENCES}

Amann RI, Krumholz L, Stahl DA. 1990. Fluorescent-oligonucleotide probing of whole cells for determinative, phylogenetic, and environmental studies in microbiology. Journal of Bacteriology 172(2):762-770 DOI 10.1128/jb.172.2.762-770.1990.

Bock C, Pröschold T, Krienitz L. 2011. Updating the genus dictyosphaerium and description of mucidosphaerium gen. nov. (trebouxiophyceae) based on morphological and molecular data1. Journal of Phycology 47(3):638-652 DOI 10.1111/j.1529-8817.2011.00989.x.

Bui TVL, Ross IL, Jakob G, Hankamer B. 2013. Impact of procedural steps and cryopreservation agents in the cryopreservation of chlorophyte microalgae. PLOS ONE 8(11):e78668 DOI 10.1371/journal.pone.0078668.

Béchet Q, Muñoz R, Shilton A, Guieysse B. 2013. Outdoor cultivation of temperature-tolerant Chlorella sorokiniana in a column photobioreactor under low power-input. Biotechnology and Bioengineering 110(1):118-126 DOI 10.1002/bit.24603.

Campa-Córdova AI, Luna-González A, Ascencio F, Cortés-Jacinto E, Cáceres-Martínez CJ. 2006. Effects of chloramphenicol, erythromycin, and furazolidone on growth of Isochrysis galbana and Chaetoceros gracilis. Aquaculture 260(1-4):145-150 DOI 10.1016/j.aquaculture.2006.06.014.

Chiu S-Y, Kao C-Y, Chen T-Y, Chang Y-B, Kuo C-M, Lin C-S. 2015. Cultivation of microalgal Chlorella for biomass and lipid production using wastewater as nutrient resource. Bioresource Technology 184:179-189 DOI 10.1016/j.biortech.2014.11.080.

Christie WW. 1994. Gas Chromatography and Lipids: A Practical Guide. Ayr: The Oily Press.

Cid A, Herrero C, Torres E, Abalde J. 1995. Copper toxicity on the marine microalga Phaeodactylum tricornutum: effects on photosynthesis and related parameters. Aquatic Toxicology 31(2):165-174 DOI 10.1016/0166-445x(94)00071-w.

Dogaris I, Welch M, Meiser A, Walmsley L, Philippidis G. 2015. A novel horizontal photobioreactor for high-density cultivation of microalgae. Bioresource Technology 198:316-324 DOI 10.1016/j.biortech.2015.09.030.

Encarnação T, Pais AACC, Campos MG, Burrows HD. 2015. Cyanobacteria and microalgae: a renewable source of bioactive compounds and other chemicals. Science Progress 98(2):145-168 DOI 10.3184/003685015X14298590596266. 
Fuller BJ, Lane N, Benson EE. 2005. Life in the Frozen State. Boca Raton: CRC Press.

Gellenbeck KW. 2012. Utilization of algal materials for nutraceutical and cosmeceutical applications-what do manufacturers need to know? Journal of Applied Phycology 24(3):309-313 DOI 10.1007/s10811-011-9722-z.

Guillard RRL, Ryther JH. 1962. Studies of marine planktonic diatoms. I. Cyclotella nana Hustedt, and Detonula confervacea (cleve) Gran. Canadian Journal of Microbiology 8(2):229-239 DOI 10.1139/m62-029.

Hubálek Z. 2003. Protectants used in the cryopreservation of microorganisms. Cryobiology 46(3):205-229 DOI 10.1016/S0011-2240(03)00046-4.

Intergovernmental Oceanographic Commission of the United Nations Educational, Scientific and Cultural Organization (IOC-UNESCO). 2010. In: Karlson B, Cusack C, Bresnan E, eds. Microscopic and Molecular Methods for Quantitative Phytoplankton Analysis. Paris: IOC Manuals and Guides.

John RP, Anisha GS, Nampoothiri KM, Pandey A. 2011. Micro and macroalgal biomass: a renewable source for bioethanol. Bioresource Technology 102(1):186-193

DOI 10.1016/j.biortech.2010.06.139.

Kearse M, Moir R, Wilson A, Stones-Havas S, Cheung M, Sturrock S, Buxton S, Cooper A, Markowitz S, Duran C, Thierer T, Ashton B, Meintjes P, Drummond A. 2012. Geneious Basic: an integrated and extendable desktop software platform for the organization and analysis of sequence data. Bioinformatics 28(12):1647-1649 DOI 10.1093/bioinformatics/bts199.

Kouzuma A, Watanabe K. 2015. Exploring the potential of algae/bacteria interactions. Current Opinion in Biotechnology 33:125-129 DOI 10.1016/j.copbio.2015.02.007.

Krienitz L, Hegewald EH, Hepperle D, Huss VAR, Rohr T, Wolf M. 2004. Phylogenetic relationship of Chlorella and Parachlorella gen. nov. (Chlorophyta, Trebouxiophyceae). Phycologia 43(5):529-542 DOI 10.2216/i0031-8884-43-5-529.1.

Lai H-T, Hou J-H, Su C-I, Chen C-L. 2009. Effects of chloramphenicol, florfenicol, and thiamphenicol on growth of algae Chlorella pyrenoidosa, Isochrysis galbana, and Tetraselmis chui. Ecotoxicology and Environmental Safety 72(2):329-334 DOI 10.1016/j.ecoenv.2008.03.005.

Lubarsky HV, Hubas C, Chocholek M, Larson F, Manz W, Paterson DM, Gerbersdorf SU. 2010. The stabilisation potential of individual and mixed assemblages of natural bacteria and microalgae. PLOS ONE 5(11):e13794 DOI 10.1371/journal.pone.0013794.

Mahapatra DM, Ramachandra TV. 2013. Algal biofuel: bountiful lipid from Chlorococcum sp. proliferating in municipal wastewater. Current Science 105:47-55.

Mara DD. 2004. Domestic Wastewater Treatment in Developing Countries. Sterling: Earthscan Publications.

Masojídek J, Torzillo G, Kopecký J, Koblížek M, Nidiaci L, Komenda J, Lukavská A, Sacchi A. 2000. Changes in chlorophyll fluorescence quenching and pigment composition in the green alga Chlorococcum. Journal of Applied Phycology 12:417-426 DOI 10.1023/A:1008165900780.

Matos ÂP, Feller R, Moecke EHS, de Oliveira JV, Junior AF, Derner RB, Sant'Anna ES. 2016. Chemical characterization of six microalgae with potential utility for food application. Journal of the American Oil Chemists Society 93(7):963-972 DOI 10.1007/s11746-016-2849-y.

Maurya R, Paliwal C, Chokshi K, Pancha I, Ghosh T, Satpati GG, Pal R, Ghosh A, Mishra S. 2016. Hydrolysate of lipid extracted microalgal biomass residue: an algal growth promoter and enhancer. Bioresource Technology 207:197-204 DOI 10.1016/j.biortech.2016.02.018. 
Müller J, Day JG, Harding K, Hepperle D, Lorenz M, Friedl T. 2007. Assessing genetic stability of a range of terrestrial microalgae after cryopreservation using amplified fragment length polymorphism (AFLP). American Journal of Botany 94(5):799-808 DOI 10.3732/ajb.94.5.799.

Prommuak C, Pavasant P, Quitain AT, Goto M, Shotipruk A. 2012. Microalgal lipid extraction and evaluation of single-step biodiesel production. Engineering Journal 16(5):157-166 DOI 10.4186/ej.2012.16.5.157.

Pruesse E, Quast C, Knittel K, Fuchs BM, Ludwig W, Peplies J, Glöckner FO. 2007. SILVA: a comprehensive online resource for quality checked and aligned ribosomal RNA sequence data compatible with ARB. Nucleic Acids Research 35(21):7188-7196 DOI 10.1093/nar/gkm864.

Rodrigues DB, Menezes CR, Mercadante AZ, Jacob-Lopes E, Zepka LQ. 2015. Bioactive pigments from microalgae Phormidium autumnale. Food Research International 77:273-279 DOI 10.1016/j.foodres.2015.04.027.

Saadaoui I, Emadi MA, Bounnit T, Schipper K, Jabri HA. 2016. Cryopreservation of microalgae from desert environments of Qatar. Journal of Applied Phycology 28(4):2233-2240 DOI 10.1007/s10811-015-0743-x.

Seoane M, Rioboo C, Herrero C, Cid Á. 2014. Toxicity induced by three antibiotics commonly used in aquaculture on the marine microalga Tetraselmis suecica (Kylin) Butch. Marine Environmental Research 101:1-7 DOI 10.1016/j.marenvres.2014.07.011.

Sharma AK, Sahoo PK, Singhal S, Patel A. 2016. Impact of various media and organic carbon sources on biofuel production potential from Chlorella spp. 3 Biotech 6(2):116 DOI 10.1007/s13205-016-0434-6.

Shiraishi H. 2016. Cryopreservation of the edible alkalophilic cyanobacterium Arthrospira platensis. Bioscience, Biotechnology, and Biochemistry 80(10):2051-2057 DOI 10.1080/09168451.2016.1189320.

Silkina A, Nelson GD, Bayliss CE, Pooley CL, Day JG. 2017. Bioremediation efficacycomparison of nutrient removal from an anaerobic digest waste-based medium by an algal consortium before and after cryopreservation. Journal of Applied Phycology 29(3):1331-1341 DOI 10.1007/s10811-017-1066-x.

Wang L, Li Y, Sommerfeld M, Hu Q. 2013. A flexible culture process for production of the green microalga Scenedesmus dimorphus rich in protein, carbohydrate or lipid. Bioresource Technology 129:289-295 DOI 10.1016/j.biortech.2012.10.062.

Wehr JD, Sheath RG (eds). 2003. Chapter 22: Brown Algae. In: Freshwater Algae of North America: Aquatic Ecology. Burlington: Academic Press, 757-773 DOI 10.1016/B978-012741550-5/50027-1.

Yang F, Xiang W, Sun X, Wu H, Li T, Long L. 2014. A novel lipid extraction method from wet microalga Picochlorum sp. at room temperature. Marine Drugs 12(3):1258-1270 DOI 10.3390/md12031258.

Zhu F, Massana R, Not F, Marie D, Vaulot D. 2005. Mapping of picoeucaryotes in marine ecosystems with quantitative PCR of the 18S rRNA gene. FEMS Microbiology Ecology 52:79-92 DOI 10.1016/j.femsec.2004.10.006. 